\title{
Input Devices to Support Communication For People with Serious Disability
}

\author{
Chao Zhang, Shunichi Kumano \\ Graduate School of Science and Technology, \\ Nagasaki University \\ Nagasaki, Japan \\ bb52311103@cc.nagasaki-u.ac.jp \\ smoromug@nagasaki-u.ac.jp
}

\author{
Kennichi Takashima, Motohiro Tanaka \\ Shunji Moromugi, Takakazu Ishimatsu \\ Graduate School of Science and Technology, \\ Nagasaki University \\ Nagasaki, Japan \\ ishi@nagasaki-u.ac.jp
}

\begin{abstract}
Persons suffering from intractable disease like ALS (Amyotrophic Lateral Sclerosis) and spinal cord injured present symptoms of muscular deteriorations. These patients are often obliged to use artificial respirators, once the breathing action becomes impossible. It is important to understand that using the artificial respirator means that uttering voice becomes impossible. Talking and discussing with friends via voice is necessary for everybody to enjoy our daily life.

In order to support their communication, there exist various input devices like touch sensor input devices, capacitance sensor input devices and also vision input devices. These input devices are developed by using the various sensors.

In this paper principles of various input devices are explained and also application techniques to patient with serious disability are shown. Applicable range of every input device are limited depend on the sensor introduced, Vision input devices are applicable to wider range of patients. But vision input devices require some knowledge about lighting conditions and basic electronics. Each input device has various features. Therefore, the user should understand the feature of the device and should use tin the proper way.

In addition to the input devices, computer controlled environment is also important. By developing proper computer controlled environment which is controlled by the proper input device, even the patient with serious disability can live sound life.

In this paper one advanced computer controlled environment which is developed for a patient with spine injury. The feature of the environment is that infrared network system is introduced. Due to this introduction, the computer controlled environment could be well organized.
\end{abstract}

Keywords-disability; assistive; communiction; computer controlled; visio sensor; input device; communication

\section{INTRODUCTION}

People should be able to enjoy their independent life. However, patients suffering from serious disease like ALS (Amyotrophic Lateral Sclerosis) or cerebral infarction are difficult to live independent lives. They need intensive cares by care-workers. As a typical symptom of ALS patients their muscular functions deteriorate rapidly. In two or three years after the onset of the disease, patients are obliged to use artificial respirators. Once they start to use the artificial respirator, uttering voice becomes impossible. In such a physical situation, they are usually confined to bed and physical ability are limited to slight movements of head, fingers, lips or eyes. Therefore, their relationships with society are extremely limited. In Fig.1 a typical ALS patient is shown who is living his house under the care of his wife and medical care staff for about 20 year However, recent development of computer technology and network technology brought great benefits to these peoples with physical disability.

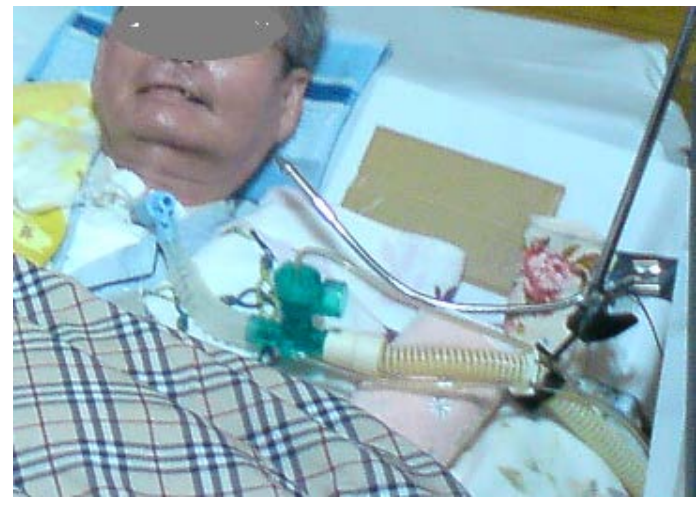

Fig.1 ALS patient with artificial respirator

Using the computer and network system, even the person with serious physical disability can communicate and talk with others in far places even if they are confined to bed. Various computer systems to support their communication skill are already commercialized. It should be noticed that one difficult problem is how to operate the computer under their serious physical conditions. Considering their deteriorated muscular ability, some input devices for various users were developed. Those input deices are categorized as a stand type and a wearable type [1]. The stand type devices include touch switches, laser sensors and vision sensors. These devices are usually attached to the bed or table and used to detect some body movements. Wearable type devices employ sensors like acceleration, angle, strain and EMG (Electromyogram) sensors that are attached to the patient's body. These sensors measure 
physical movement or condition of the patient body and input signal is generated based on the data measured.

In the followings various kinds of input devices are shown. Touch sensor is a typical conventional device. Slight touch by the human body and human finger onto the sensor head can be detected by the change of amplitude of sinusoidal elastic noise generated by the commercial power line near bye. The principle is shown in Fig.2.

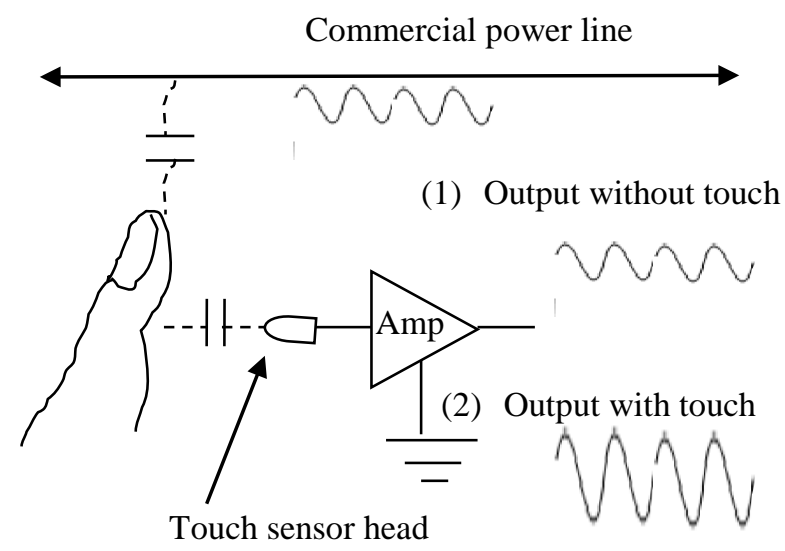

Fig.2 Touch sensor output generated by commercial power line

If human body touches the sensor head, the amplitude of generated sinusoidal noise is amplified as shown in Fig.2. Considering this feature of the touch sensor, the touch sensor can be applied to other type of input sensors as shown in the next chapter.

Even the touch sensor can be used in the various ways as input switch. Of course, other sensors can be used as effective input devices. For example, the sensors are pressure sensor, acceleration sensor, miniature rotary encoder, eye electric potential and so on.

It should be noticed that the input device to use vision have distinguished advantages over the other devices since the vision devices have less interference with human body and can be applicable to person with serious physical disability [2], [3]. The vision device can be settled apart from the bed. This means care workers can be free from annoying setting task, wires and mechanical parts around the bed. Another advantage is that the vision device can be applied to detect various body movements like lips, fingers, mouth, eyebrow as well as eyeball. It is also important to note that the vision devices can be applicable to various physical ability of the user. In the first stage of the intractable disease like ALS the patient can move his head. But after the physical situation becomes serious, he sometimes can move his eyeball or eyebrow. Of course, the situation depends on the patient. A feature of the vision device is that the vision device is readily applicable to various physical situations by renewing the image processing software.

In this paper examples of the developed input devices those are applied to serious patients are demonstrated. These devices are incorporated into the computer system to support communication of the patient. Applicability of our proposed input devices is discussed.

\section{INPUT DEVICES TO USE TOUCH SENSOR}

Principle of the touch sensor can be readily applicable to the following two cases.

\section{A. Ring type touch switch}

In Fig. 3 a ring type switch is shown which is developed for the user suffered by cerebral infarct. The inner surface of the ring is covered by non-conductive material. Only outer and thumb finger side has conductivity. Therefore, only the slight movement of the finger is enough to activate the touch switch. Using this sensor the patient succeeded to communicate with his wife for more than couple of years.

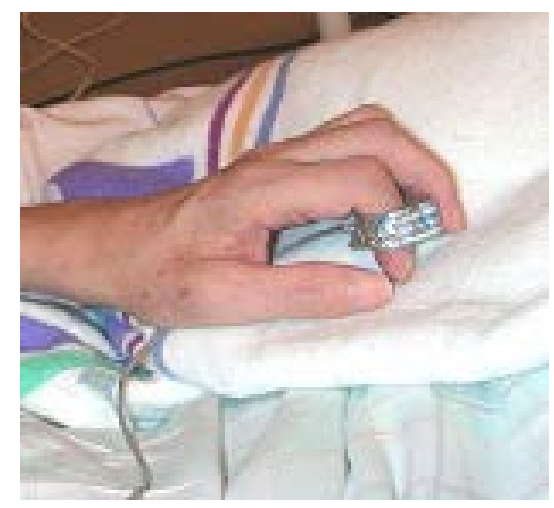

Fig.3 Ring sensor used by the user suffered by cerebral infarct

\section{B. Rod type touch switch}

In Fig. 4 a rod type touch switch settled above the bed is shown. The surface of the rod is covered by the conductive material. This switch is requested by a user suffered by brain paralysis. He couldn't move his upper and lower limbs. But he could lift his head and touch the rod by his forehead. This input device is used to activate emergency call.

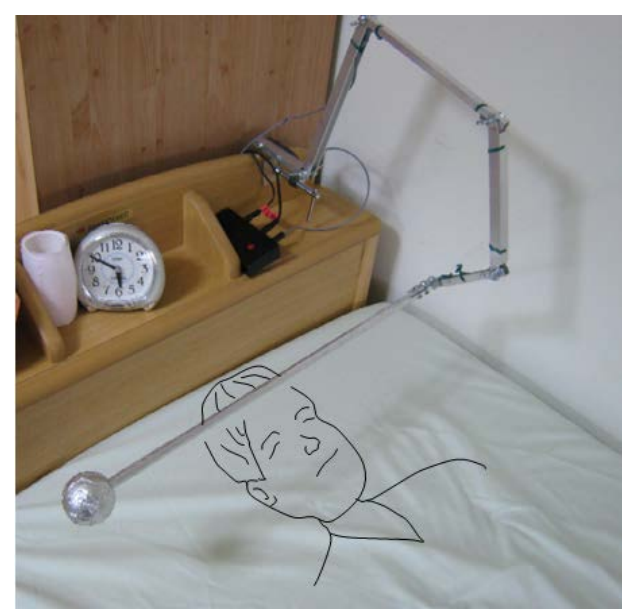

Fig.4 Rod type touch switch 


\section{INPUT DEVICE TO DETECT ELECTRICAL CAPACITY}

Input devices to detect electrical capacity are often effective as shown in the followings.

\section{A. Grip type capacitance switch}

In Fig. 5 a grip type switch is shown which is developed for an ALS patient. The cross section of the grip switch is shown in Fig.6. Around the cylindrical conductive rod non-conductive soft material is surface covered. And outer surface of the grip switch is covered by conductive soft cloth.

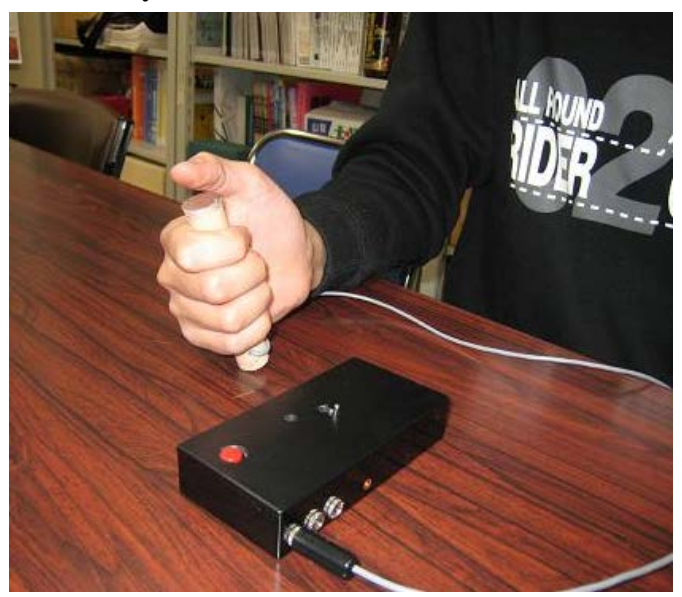

Fig.5 Grip switch developed for ALS patient

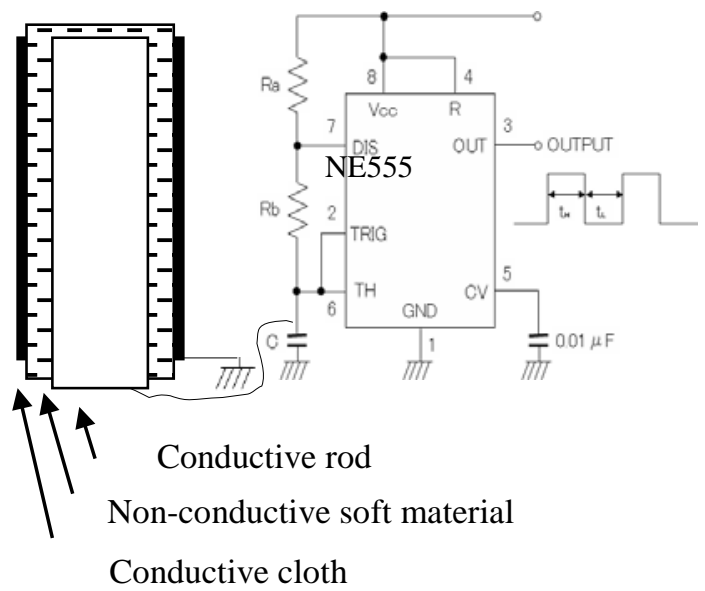

Fig.6 Cross-section of the grip switch

In Fig.6 one typical circuit to measure electrical capacitance is shown. The capacitance can be measured by the frequency of the output signal. Due to this constitution of the switch the serious patient could use the device an effective switch for the emergency call.

\section{B. Flat type capacitance switch}

In Fig.7 flat type capacitance switch developed for a patient suffered by rheumatism is shown. The switch was successfully applied to the rheumatism patient while the user could move

\footnotetext{
Identify applicable sponsor/s here. If no sponsors, delete this text box (sponsors).
}

her upper limbs inside limited area above the chest. It is important to note that some serious patients often have impaired sensation.

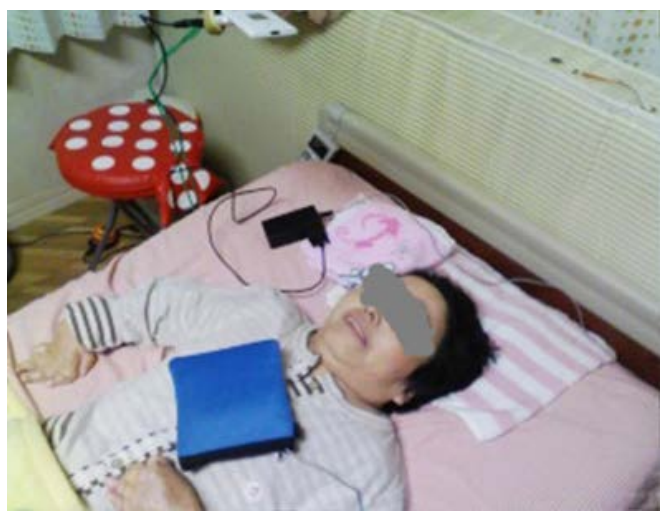

Fig.7 Flat type capacitance switch on the chest

This means such user often fails to manipulate the switch. However, if the user has healthy sensory capability around his chest, this flat type capacitance sensor can be used effectively. The sensory signal around chest reveals the situation of the operation of the flat type switch. This switch was applied to two patients suffered by cerebral infarct successfully.

\section{INPUT DEVICE TO USE ROTARY ENCODER}

ALS disease is admitted as a serious intractable disease. According to the progress of the symptom, the patient reveals various physical impairments. The ALS patient shown in Fig.8 could move his muscles around eyebrows slightly. An input device to detect muscle movements using the rotary encoder was developed for such patient.

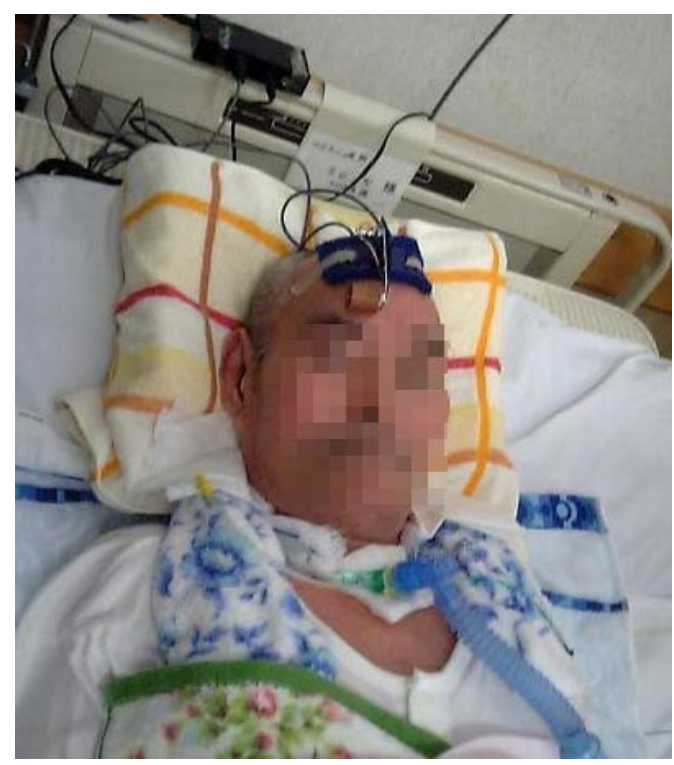

Fig.8 Input device to use rotary encoder 
The configuration of the switch is shown in Fig.9. Using the rotary encoder with high-resolution, muscle movements can be detected with accuracy.
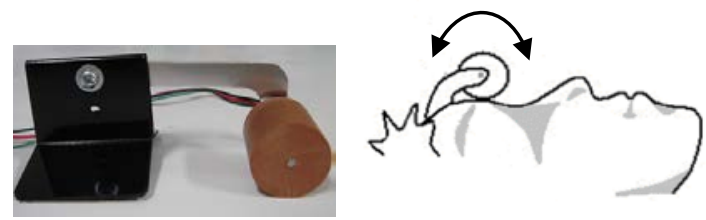

Fig.9 Configuration of input device

\section{INPUT DEVICE TO USE BENDING SENSOR}

The bending sensor is able to detect the finger movement easily. In Fig.10 the bending sensor is attached to the thumb finger.

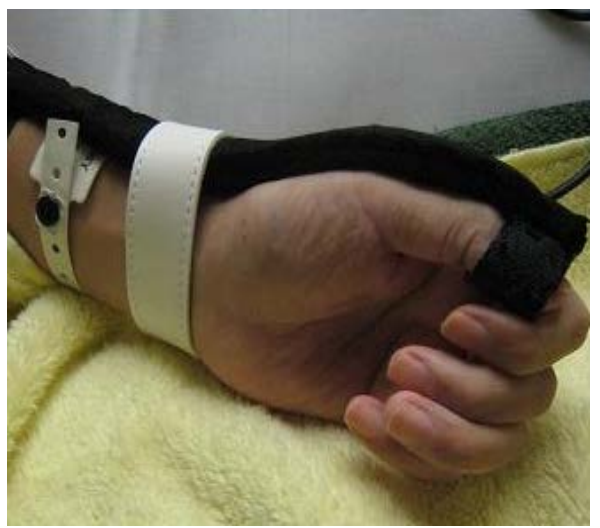

Fig.10 Bending sensor attaché to thumb finger

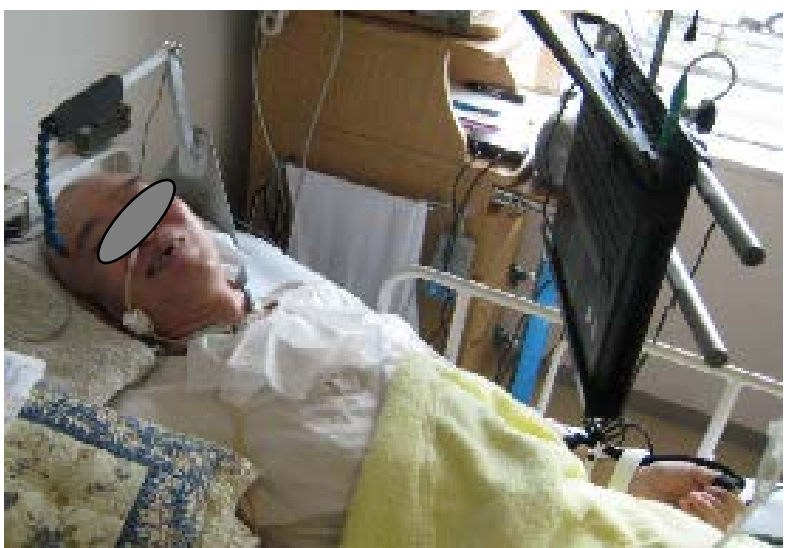

Fig.11 Patient with bending switch

Fig.11 shows a patient suffered by cerebral infarct operating a computer using our bending switch. Near his head, an emergency call to use touch switch is mounted. Using the emergency call and the computer system, he could live enjoying the communication with family and care-workers.

\section{INPUT DEVICE TO USE CHEWING ACTION}

In order to enable simple and fast operations of the computer, two-dimensional pointing ability is inevitable. And also simple switching ability to enable click-button operation is necessary. Two-dimensional pointing ability can be achieved by employing two-dimensional acceleration sensor. Fast switching operation can be realized by using the chewing action. Chewing action can be detected by measuring the swelling situation of the temporal muscle. The place of temporal muscle is shown in Fig.12. It can be readily recognized that chewing action the temporal muscle contracts and the position.
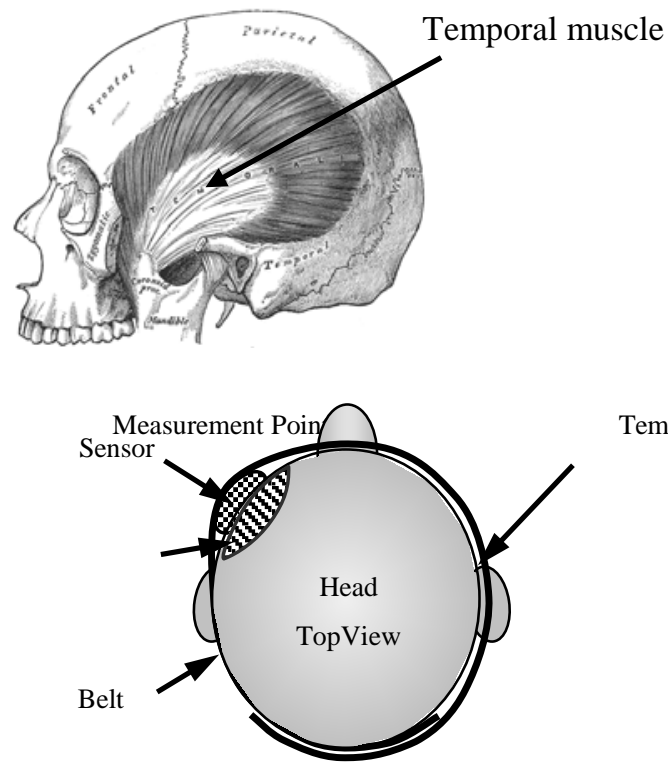

Temporalis

Fig. 12 Installation of sensor

We focused on the temporal muscle as the measurement portion. Based on our technique the capacitive pressure sensor is touched near the temple and is fixed by the head belt as shown in Fig.12. The head belt is non-elastic and can be used just by lightly wrapping around the head.

In Fig.13 output signal of capacitive pressure sensor is shown where chewing signal can be recognized. It can be also recognized that effect of the talking action is negligible small.

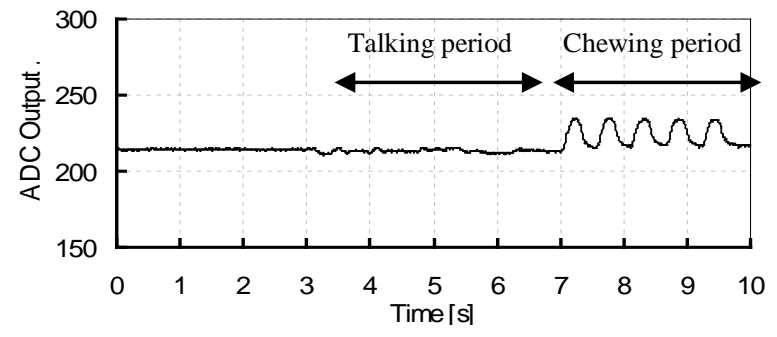

Fig.13 Output signal from capacitive pressure sensor

It is readily recognized that the number of chewing action is countable from the signal obtained by the capacitance sensor. 
This input device can be applied to an input device for the assistive device for a user with spinal injury. The user's upper limbs are impaired. In Fig.14 the user is wearing assistive glove which enables finger actions by using DC motors. In the system the chewing action is employed as efficient input switch. He conducted the pick and place test using the proposed system. The test result was satisfactory.

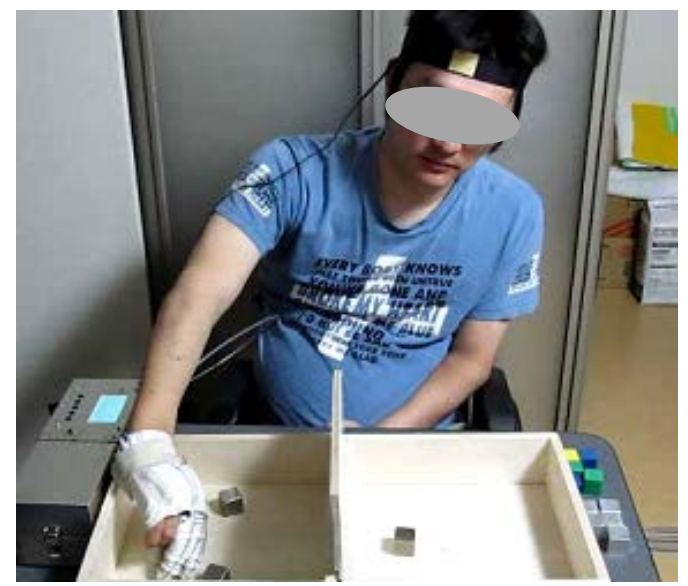

Fig.14 Pick and place test using assistive glove

Nowadays dentists employ the number of chewing actions during the meal as one important index to evaluate the soundness. Therefore, effective counting device is required. In Fig.15 developed chewing counter is proposed which is composed of a measuring unit and a capacitive pressure sensor. The capacitive pressure sensor is attached to temporal muscle by the head belt.

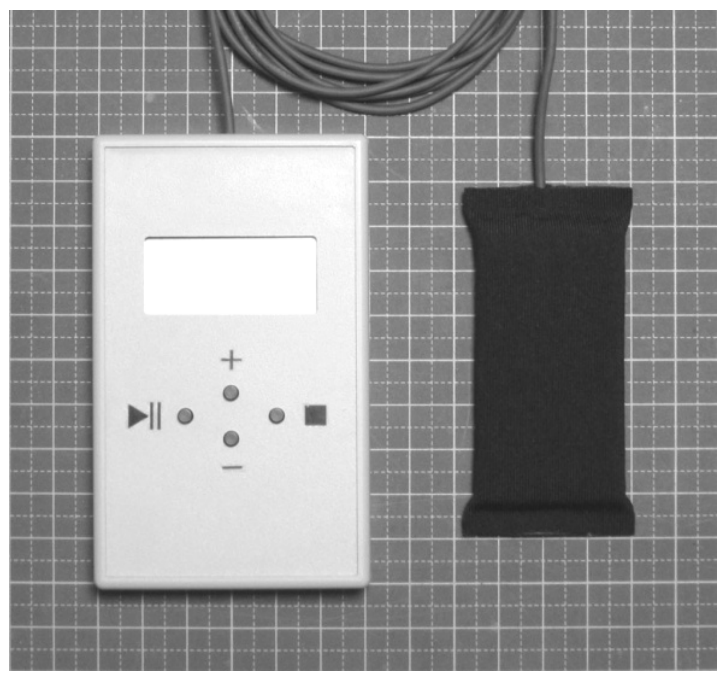

Fig.15 Chewing counter

Dental health survey to use our original chewing counter was conducted in Nagasaki city. The survey revealed the results shown in Table 1 . The data were obtained by 29 females and 63 males.

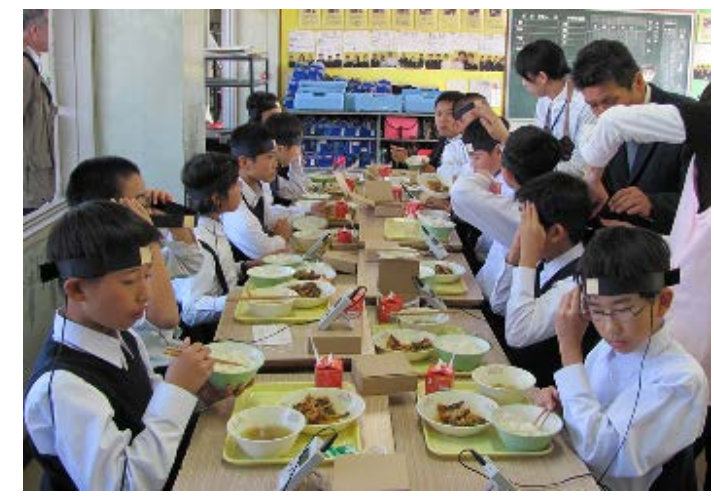

Fig.16 Dental health survey

\begin{tabular}{|} 
Table 1 Result of dental health survey \\
\begin{tabular}{|l|c|}
\hline & Average No. chewing \\
\hline At breakfast & 819 \\
\hline At lunch & 1,001 \\
\hline At dinner & 1,700 \\
\hline In one day & 3,506 \\
\hline
\end{tabular}
\end{tabular}

\section{INPUT DEVICE TO USE ACCELERATION SENSOR}

Acceleration sensor is effective for input device for people with serious disability.

\section{A. Input device to use wrist movement}

The acceleration sensor shown in Fig.17 can detect slight movements of the hand in two directions. Therefore, this sensor can be applicable to move the computer cursor in two dimensionally.

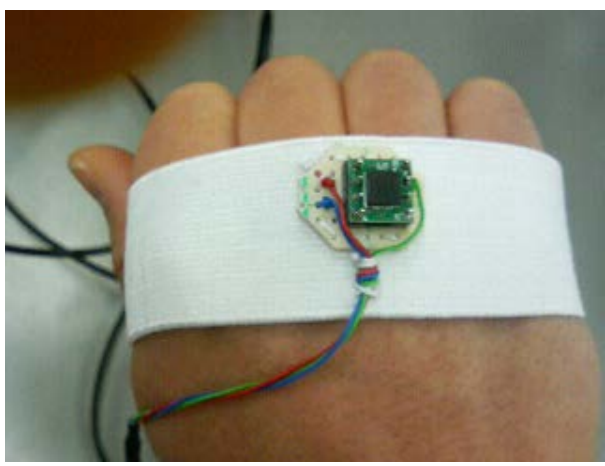

Fig.17 Input device to use wrist movement

\section{B. Pointing device to use head movement}

In Fig.18 a patient with spine injury is shown. Because of the injury, his physical ability was limited only above his neck. Furthermore, he had communication disability. After the acute hospitalization period, his desire was to recover communication ability. In Fig.18 our original input device was shown, where an acceleration sensor is mounted on the band attached on the forehead. The computer cursor in the computer 
display can be moved by the head movement of the user. Click operation can be achieved by the chewing action.

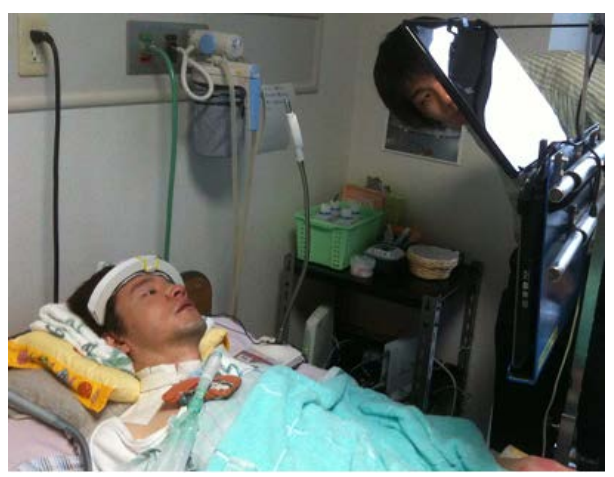

Fig.18 Input device for user with spine injury

Using our proposed input device, the user could communicate with others and exchange e-mail with friends.

\section{INPUT DEVICE TO USE EOG SIGNAL}

EOG (Electro-Oculogram) signal can be used to detect the direction of the eye ball. The EOG signal can be readily obtained by the differential signal obtained at both edge of two eyeballs as shown in Fig.12.

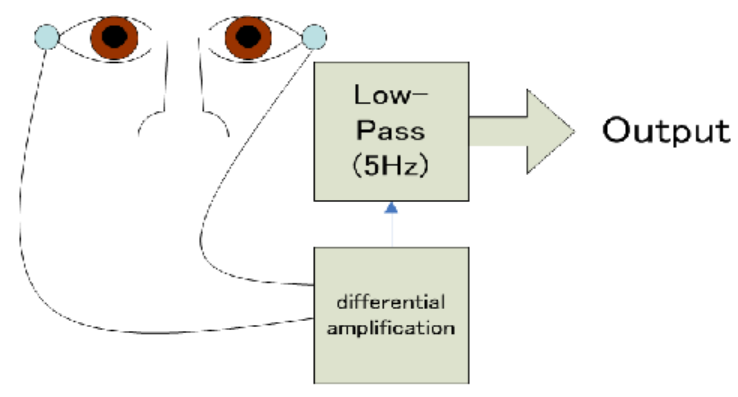

Fig.12 Principle to obtain EOG signal

In case of serious ALS patient, the remaining physical ability is often limited only at eyeballs. In such case the EOG signal is one possible way remained. In Fig.13 an ALS patient is using the input device to use EOG signal. This device is used to control TV channel and power switch.

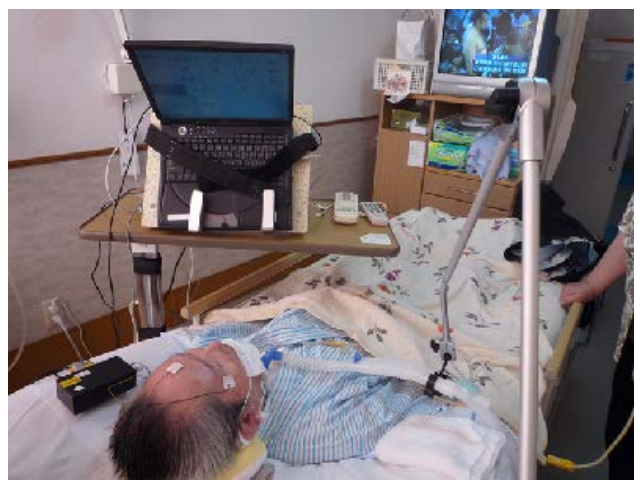

Fig.14 Input device to use EOG signal

\section{INPUT DEVICE TO USE VISION}

Input devices to use vision have distinct feature over the other input devices. One distinguished feature is that the vision sensor can be settled apart from the user. Furthermore, the vision information gives us useful information like movement of eyeball and head movements and also other muscle movement around the body. In Fig.15 an input device to use vision is shown where one camera and display monitor are mounted above the bed.

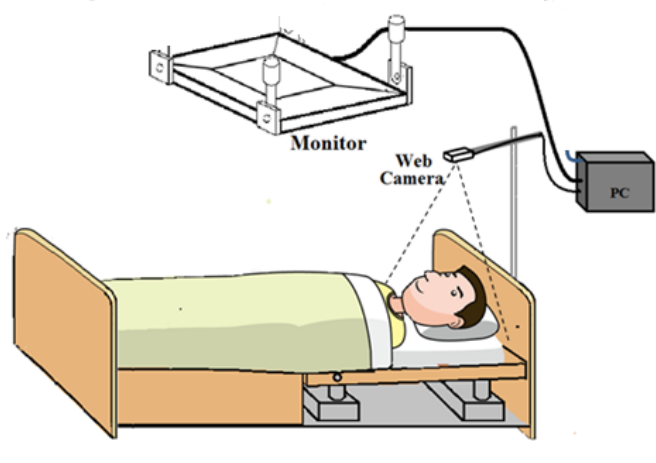

Fig.15 Vision system and the patient

There are some image processing software which is applicable as input device. One robust image processing software even under the unfavorable lighting condition is to use color marks on the face as shown in Fig.19.

By introducing the color marks, the head movements can be detected accurately. Furthermore, by introducing the second color mark on the chin, the opening action of the mouth can be detected. Opening action of the mouth can be effectively used as click action. Therefore, only by the face movements the computer can be operated.

Of course, the image processing without the color mark is more favorable.

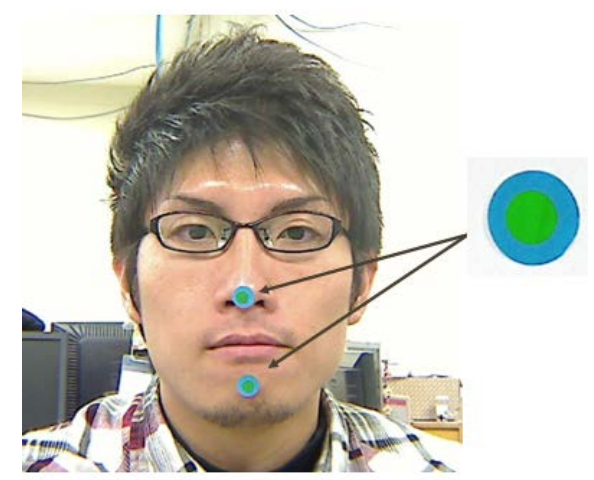

Fig.19 Face image with color marks 


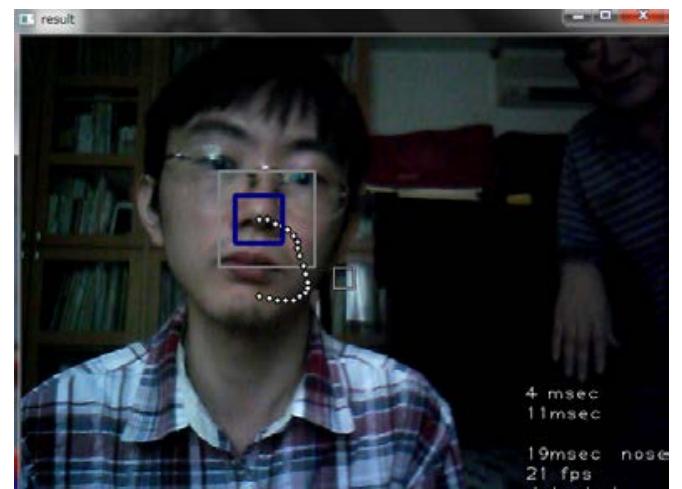

Fig.20 Trajectory of the nose movement

In Fig.20 the image around the nose is focused and by using the pattern matching technique the face movement is traced. In Fig.20 the result of the trajectory of the nose movement is displayed. Deformation of the nose image caused by the face during face movement could be compensated. The computer cursor can be controlled according to the nose movement.

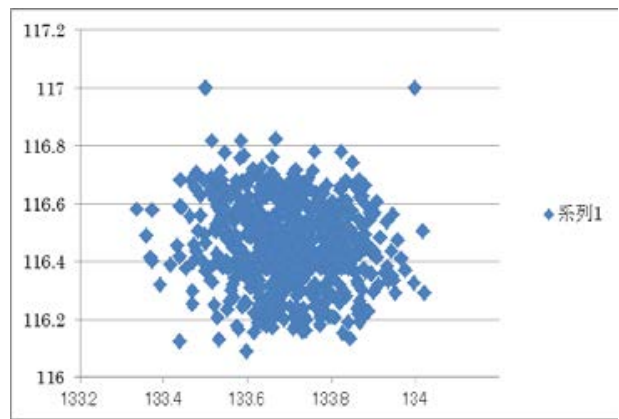

Fig.21 Obtained raster coordinate of the nose

In order to move computer cursor accurately, sub-pixel image processing is preferable. Fig. 21 shows results of the subpixel image processing under the condition that nose positions are fixed at definite position. In the horizontal and vertical directions position data are distributed in the range of 0.6 pixels.

Pointing accuracy during tracking task by the head movement was evaluated by healthy examinees without any physical disability. The data obtained by the pointing device were used to locate the mouse pointer on the computer display in proportion to the head movement. A camera (CCD sensor with $570 \mathrm{H} \times 480 \mathrm{~V}$ resolution) was settled $200 \mathrm{~cm}$ apart from the examinee. The examinee was requested to move his head so that the mouse pointer traces the rectangular shape in the computer display. And also the examinee was requested to conduct click action at all four corners. The click action means opening his mouse. The width and height of the target rectangle on the computer display was 500 pixels as shown in Fig.22.

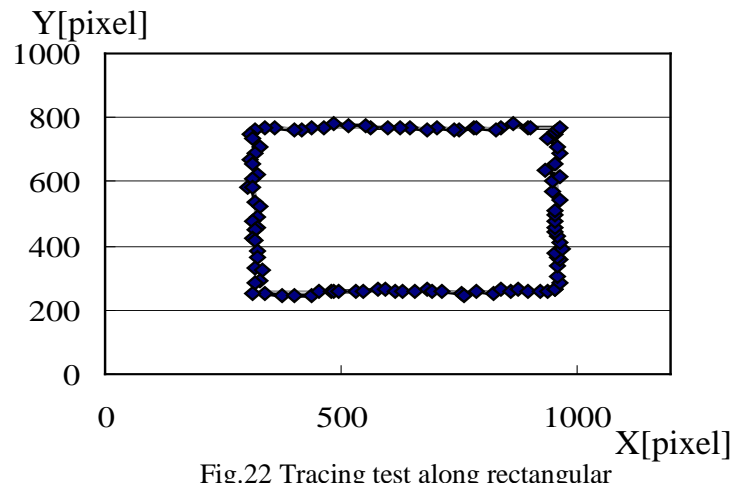

The vision input device was applied to input text data. A computer display of the communication software is shown in Fig.23. In the lower half of this display many buttons were arranged. To each button one character or one function was allocated.

By selecting the desired button sequentially by the head movement and mouth movement, desired text could be completed as shown in the upper half of the display.

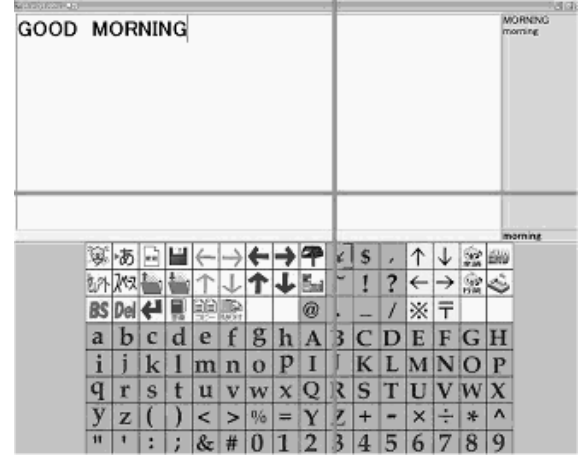

Fig.23 Computer display of communication system

Table 2 shows time required to write the message "GOOD MORNINNG”. Conventional technique to use touch sensor ways required more than three minutes.

Table 2 Time required for writing message

\begin{tabular}{|c|c|c|c|c|c|}
\hline $\begin{array}{c}\text { User } \\
\text { No. }\end{array}$ & 1 & 2 & 3 & 4 & 5 \\
\hline $\begin{array}{c}\text { Trial 1 } \\
\mathrm{T}=0.5 \mathrm{~s}\end{array}$ & 26 & 28 & 22 & 29 & 26 \\
\hline
\end{tabular}

\section{COMPUTER CONTROLLED ENVIROMENT}

In Fig.24 a computer controlled environment is shown where the patient with spinal injury uses a vision input device and many kinds of electric appliances are controlled via infrared signals. Controlled electric appliances are ceiling 
lights, air-conditioning system, emergency call, telephone, TV and so on. A distinct function introduced here is that that the posture and position of the TV monitor can be adjusted by the computer commands via infrared signal since the patient is requested to change his posture on the bed at every two hours to prevent the bedsore. Therefore, TV is mounted on the ceiling with original mechanism. By introducing the infrared network system, the environment around the bed is well organized.

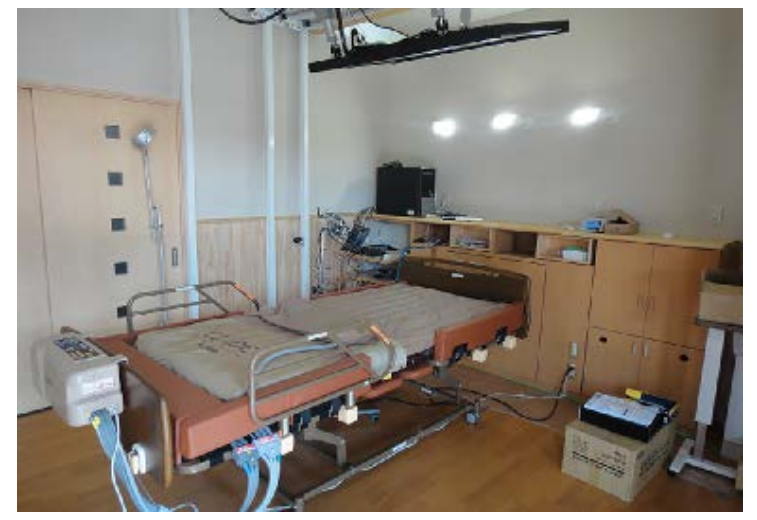

Fig.24 Advanced computer controlled environment via infrared network

In order to achieve effective computer controlled system, a compact infrared emitter and a receiver was developed as shown in Fig.25. The emitter can send modulated infrared signals according to the command signals obtained. The receiver can receive the modulated infrared signals and control on/off function of the relay circuit inside the receiver. Of course, the emitter signal is compatible with commercialized infrared signals.
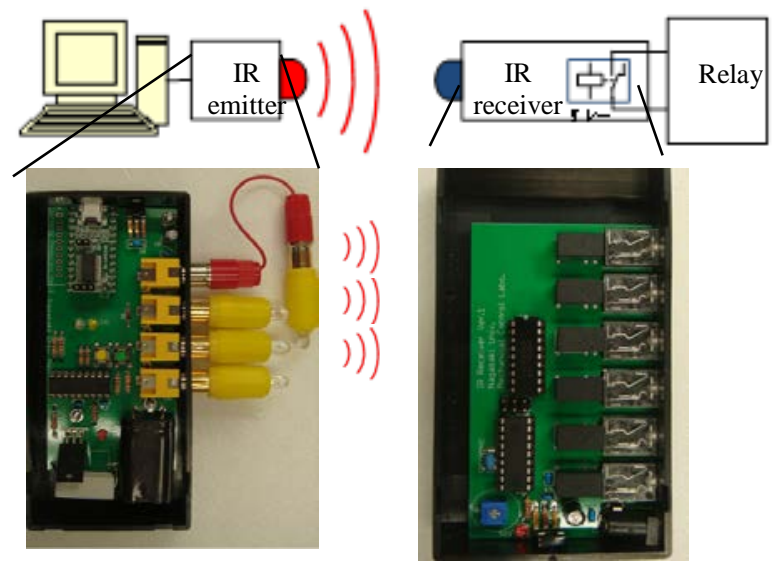

Fig.25 Infrared emitter and receiver

\section{CONCLUSIONS}

Patients with serious disease like ALS are living in serious condition. While they often have smart brains and sensory functions, their muscle functions deteriorate sooner and will be annoyed by communication disability. It is important to note that patients have various symptoms based on the physical condition. Considering the symptom and physical condition, the input devices and assistive devices should be developed. In this paper, many kinds of input devices are explained. Some of them use conventional technologies and some use advanced technologies like image processing. In the Table 3, proposed switches are compared. While applicable physical abilities are limited depend on the input devices, the applicable abilities are required to be wider.

Table 3 Input devices and applicable physical ability

\begin{tabular}{|l|l|}
\hline Proposed switch & Applicable physical ability \\
\hline Touch sensor switch & Finger, Head, Mouth, \\
\hline Ring sensor switch & Finger \\
\hline Grip sensor switch & Hand \\
\hline Flat type switch & Hand \\
\hline Rotary encoder switch & Eyebrow, \\
\hline Chewing switch & Chewing \\
\hline Acceleration switch & Hand, Head \\
\hline EOG switch & Eyeball \\
\hline Vision switch & $\begin{array}{l}\text { Head, Hand, Eyeball, } \\
\text { Mouth, Eyebrow }\end{array}$ \\
\hline
\end{tabular}

A vision input device is recognized as an effective input device since the device is applicable to wider range of physical abilities. While some vision input devices are commercialized, there still remain some points to overcome like lighting condition, resolution, and operability. Sooner compact and advanced cameras will be developed. The advanced camera will offer smart input devices to the patients with serious disability.

\section{References}

[1] Y.Fukuda, M.Tanaka, S. Moromugi and T. Ishimatsu, A Communication device for disabled slight movement capabilities, International Journal of HWRS, vol.4, No.4, December, pp.7-12, 2003.

[2] T. Ochiai, T. Ishimatsu, Computer Input device for handicapped using vision sensor, in Proc.3rd Int. symposium on artificial life and robotics Vol.2, pp.634-637, 1997.

[3] O.Takami, T.Ishimatsu, and T.Shimomachi, Development of the environmental control system by using eyeball movements, Proceeding of the Asian Control Conference, pp.715-718, July 27-30, 1994.

[4] O.Takami, K.Morimoto, T.Ochiai, and T. Ishimatsu, Computer Interface to use head and eyeball movement for handicapped people, 1995 IEEE International Conference of System, Man and Cybernetics, Vancouver, Canada, October 22-25, 1995.

[5] H.Kawamura, N.Dohi, T.Oshima, "Experimental Production of Light Mouse using PSD sensor", Proc. of 5th Rehabilitation Engineering, Tokyo, Japan, 21-24 August, 1990, pp.233-236 